\title{
Hybrid Decoder with an Adaptive Fuzzy Logic Filter for Single-User Millimeter Wave Systems Under Impulsive Noise
}

\section{Mustafa Mulla ( $\sim$ mustafa.mulla@emu.edu.tr)}

Eastern Mediterranean University: Dogu Akdeniz Universitesi https://orcid.org/0000-0003-4000-5407 Ahmet Rizaner

Eastern Mediterranean University: Dogu Akdeniz Universitesi

Ali Hakan Ulusoy

Eastern Mediterranean University: Dogu Akdeniz Universitesi

\section{Research Article}

Keywords: Mixture noise model, impulsive noise, fuzzy logic, millimeter wave, massive multiple-inputmultiple-output

Posted Date: June 1st, 2021

DOI: https://doi.org/10.21203/rs.3.rs-364822/v1

License: (c) (1) This work is licensed under a Creative Commons Attribution 4.0 International License. Read Full License 


\title{
Tittle Page
}

Title: Hybrid Decoder with an Adaptive Fuzzy Logic Filter for Single-User Millimeter Wave Systems under Impulsive Noise

\section{Authors' names:}

\section{"Mustafa Mulla" (Corresponding Author)}

mustafa.mulla@emu.edu.tr

Department of Electrical and Electronic Engineering, Eastern Mediterranean University, 99628, Famagusta, North Cyprus via Mersin 10, Turkey

\author{
"Ahmet Rizaner" \\ ahmet.rizaner@emu.edu.tr \\ Department of Information Technology, Eastern Mediterranean University, 99628, \\ Famagusta, North Cyprus via Mersin 10, Turkey
}

\author{
"Ali Hakan Ulusoy" \\ alihakan.ulusoy@emu.edu.tr \\ Department of Information Technology, Eastern Mediterranean University, 99628, \\ Famagusta, North Cyprus via Mersin 10, Turkey
}

\begin{abstract}
The conventional millimeter wave systems are mostly designed to operate only for the Gaussian noise model. Although this model simplifies the noise removal problem, recent research findings state that a mixture noise model with additive impulsive noise is a more realistic approximation for millimeter wave channels. In this paper, we propose a novel approach to eliminate the impulsive noise effects on single-user millimeter wave massive multiple-input-multiple-output system using an adaptive fuzzy logic filter. Hence, a fuzzy median filter is applied to the system and it is aimed to minimize the effects of the impulsive noise by ordering samples based on fuzzy rank. Simulation results show that the proposed filter successfully eliminates the impulsive noise effects and achieves a better bit error rate and spectral efficiency performance than the competing methods in the literature while also working efficiently in Gaussian noise.
\end{abstract}

Keywords: Mixture noise model, impulsive noise, fuzzy logic, millimeter wave, massive multiple-input-multiple-output 


\section{Declarations}

- Funding

Not applicable.

- Conflicts of interest/Competing interests

The author(s) declare(s) that they have no competing interests.

- Availability of data and material

The data that support the findings of this study are available from the corresponding author, upon reasonable request.

- Code Availability

Code is available from the corresponding author, upon reasonable request.

- Authors' contributions

All authors contributed to the study conception and design. Material preparation, data collection and analysis were performed by Mustafa Mulla, Ahmet Rizaner and Ali Hakan Ulusoy. The first draft of the manuscript was written by Mustafa Mulla and all authors commented on previous versions of the manuscript. All authors read and approved the final manuscript. 


\title{
Hybrid Decoder with an Adaptive Fuzzy Logic Filter for Single-User Millimeter Wave Systems under Impulsive Noise
}

\begin{abstract}
The conventional millimeter wave systems are mostly designed to operate only for the Gaussian noise model. Although this model simplifies the noise removal problem, recent research findings state that a mixture noise model with additive impulsive noise is a more realistic approximation for millimeter wave channels. In this paper, we propose a novel approach to eliminate the impulsive noise effects on single-user millimeter wave massive multiple-input-multiple-output system using an adaptive fuzzy logic filter. Hence, a fuzzy median filter is applied to the system and it is aimed to minimize the effects of the impulsive noise by ordering samples based on fuzzy rank. Simulation results show that the proposed filter successfully eliminates the impulsive noise effects and achieves a better bit error rate and spectral efficiency performance than the competing methods in the literature while also working efficiently in Gaussian noise.
\end{abstract}

Keywords: Mixture noise model, impulsive noise, fuzzy logic, millimeter wave, massive multipleinput-multiple-output

\section{Introduction}

Mobile broadband communication requires a dramatic growth in the existing network capacity. Since there is a crucial increase in the number of users due to the rising demand for the smart system applications, the network capacity should be improved to serve the needs of the users. The researchers are predicting that the traffic load will increase approximately 1000 times in the upcoming decade and the existing $4^{\text {th }}$ Generation $(4 \mathrm{G})$ network is not enough to deal with the huge demand $[1,2]$. Thus, a new generation network called the $5^{\text {th }}$ Generation $(5 \mathrm{G})$ needs to be employed for a new spectrum with much higher bandwidth. The promising candidate to achieve such higher bandwidths is the millimeter wave (mmWave) communication with the available spectrum between $3 \mathrm{GHz}(\lambda=100 \mathrm{~mm})$ and 300 $\mathrm{GHz}(\lambda=1 \mathrm{~mm})$. It is a fact that the available frequency band on mmWave is higher than all the licensed spectrum used by the current wireless communication systems [3-5].

The capacity of the system can be increased further using the advanced antenna and diversity techniques, such as massive multiple-input-multiple-output (MIMO) [6, 7]. In the conventional MIMO, precoding is implemented at baseband using fully digital precoders. However, digital precoders require a dedicated RF chain with the signal mixers and analog-to-digital converters for each antenna element and this will increase the power consumption and the complexity of the system significantly. Therefore, hybrid analog/digital precoding is proposed by the researchers to reduce the power consumption and hardware cost of the system, since the hybrid precoders demand a very small number of RF chains compared with the number of antenna elements used in massive MIMO [8]. Besides the advantages of mmWave, there are some handicaps in mmWave which is not well addressed, such as the suppression of the noise.

In the literature, noise is generally modeled using Gaussian distribution to simplify the noise suppression problem. However, it is observed that the noise present in the mmWave frequencies may act non-Gaussian. This noise model can be expressed by a mixture model of Gaussian Noise (GN) and Impulsive Noise (IN). The main source of IN is considered as man-made and nearby devices often cause additive IN to the receivers. Since 5G technology requires an ultra-dense cellular network and machineto-machine communication is growing rapidly, the receivers are expected to be affected by a mixed noise rather than the classical White GN expressed in most of the research papers. In addition, atmospheric and solar static signals caused by the sunspots and thunderstorms can also be represented by IN and it is expected to degrade the communication quality in mmWave bands $[9,10]$. Recently, great research interest is growing to model IN behavior and it is observed that the Middleton Class A model [11] is a widely accepted and realistic model to express the mixture noise model for wireless communication channels [12-15]. Besides, it is shown that the presence of IN is affecting the performance of the system negatively for the applications operating in the mmWave frequencies [1620] and the degradation of the performance of massive MIMO systems under IN is investigated in [21]. 
Thus, a sophisticated method should be developed to detect and mitigate the effects of IN in mmWave massive MIMO systems.

To enhance the performance of the systems under the effects of IN, several detectors have been designed and most of the works are based on clipping and blanking [22-24]. Although these methods are simple to implement, Bit Error Rate (BER) performances of the systems are not suitable for practical use in mmWave. In [25], a threshold mechanism is developed for the detection of the impulses and the performance of the system is improved with the use of an optimal threshold. However, the performance of the system is not still well enough for practical use and there is a need for a more sophisticated method. In this manner, neural networks and deep learning algorithms are evaluated to improve the performance of the IN filters [26-28].

Another powerful approach to eliminate the IN effects is the fuzzy logic-based algorithm proposed in [29] and it is found out that this statistical method can fulfill our needs. This method aims to order the samples with fuzzy order and then eliminates the effects of IN using a fuzzy median filter. This algorithm is working well for IN channels but the performance is expected to degrade when the noise is distributed as Gaussian. Therefore, a threshold mechanism is built in [30] to detect the impulses and a fixed threshold is set to satisfy both impulsive and Gaussian scenarios. To improve the performance of the system further, an adaptive threshold mechanism is built in [31] and the filter is only applied on the detected impulses. The optimal threshold is found adaptively using the statistical measures, median, and standard deviation. It can be seen here that the threshold applied performance of the system in the Gaussian environment is much better than the system without any threshold.

In this paper, we propose a hybrid decoder with an adaptive fuzzy logic-based filter to suppress the effects of IN on mmWave communication. The fuzzy logic-based filters are employed before on microwave systems, however, there is no implementation on mmWave systems. Therefore, we adapted the fuzzy logic algorithm to mmWave massive MIMO output system with hybrid decoding and a novel threshold mechanism is applied to detect the IN samples. A modified Z-score suggested in [32] is used to detect the outlier samples and it is observed that the proposed decoder with the filter that selects a proper threshold is suitable for GN and IN environments. Simulation results illustrate that the proposed decoder with an adaptive fuzzy logic filter suppresses the effect of IN successfully while also working efficiently in GN and achieves a better BER and spectral efficiency performance than the blanking and clipping methods developed in [25].

The rest of the paper is organized as follows. Section 2 provides a description of the system model of the mmWave massive MIMO system. Section 3 summarizes the proposed method based on fuzzy logic. Section 4 demonstrates the simulation results and finally, the research is concluded in section 5.

Notations: Throughout this paper, matrix and vector definitions are denoted as bold upper-case and bold lower-case letters, respectively. Inverse, transpose, and Hermitian transpose are represented using the superscripts ${ }^{-1, T}$, and ${ }^{H}$ respectively. $\mathbb{E}[$.$] refers to the expectation, det (.) is the determinant of a$ matrix, and $\mathbb{C}$ represents the set of complex numbers. $\|.\|_{F}$ denotes the Frobenius norm, o refers to the elementwise multiplication, and $\mathbf{I}_{N}$ is used to define an identity matrix with the size $N \times N$. Real and imaginary parts of a complex matrix or vector are shown as $\operatorname{Re}[$.$] and \operatorname{Im}[$.$] , respectively.$
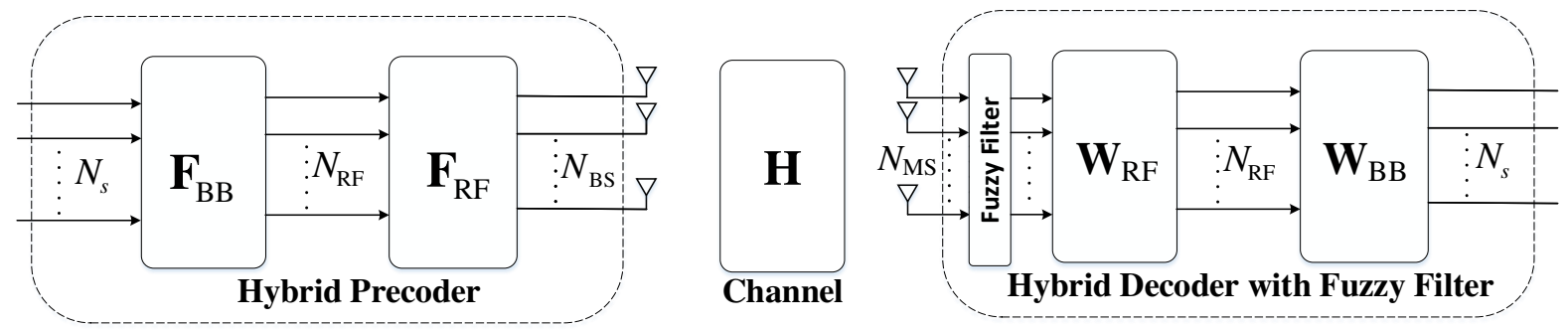

Fig. 1. System model for hybrid analog/digital architecture with a fuzzy filter in single-user mmWave massive MIMO system.

\section{System Model}

Fig. 1 illustrates the system model for a single user mmWave hybrid massive MIMO system with a fuzzy filter at the receiver. It is considered that a Base Station (BS) having $N_{\mathrm{BS}}$ antennas transmits $N_{\mathrm{S}}$ symbols to a single Mobile Station (MS) operating with $N_{\mathrm{MS}}$ antennas. For simplicity, both the BS and 
the MS assumed to have $N_{\mathrm{RF}} \mathrm{RF}$ chains with the constraint $N_{\mathrm{S}} \leq N_{\mathrm{RF}} \leq N_{\mathrm{MS}, \mathrm{BS}}$. Since we focused on the downlink model, the transmitted signal can be written as $\mathbf{x}=\mathbf{F}_{\mathrm{RF}} \mathbf{F}_{\mathrm{BB}} \mathbf{S}$ in which baseband precoder $\mathbf{F}_{\mathrm{BB}} \in \mathbb{C}^{N_{\mathrm{RF}} \times N_{\mathrm{S}}}$ and analog precoder $\mathbf{F}_{\mathrm{RF}} \in \mathbb{C}^{N_{\mathrm{BS}} \times N_{\mathrm{RF}}}$ are applied respectively to the transmitted symbol vector $\mathbf{s} \in \mathbb{C}^{N_{\mathrm{s}} \times 1}$ such that, $\mathbb{E}\left[\mathbf{s}{ }^{H}\right]=\mathbf{I}_{N_{\mathrm{S}}} / N_{\mathrm{S}}[8,33,34,35]$. The hybrid precoder is normalized to satisfy the power constraint $\left\|\mathbf{F}_{\mathrm{RF}} \mathbf{F}_{\mathrm{BB}}\right\|_{F}^{2}=N_{\mathrm{S}}$ and thus, the received signal observed by the MS can be expressed as

$$
\mathbf{r}=\sqrt{\rho} \mathbf{H F}_{\mathrm{RF}} \mathbf{F}_{\mathrm{BB}} \mathbf{s}+\mathbf{n},
$$

where $\rho$ is the average received power, channel matrix between the BS and the MS is denoted as $\mathbf{H} \in \mathbb{C}^{N_{\mathrm{MS}} \times N_{\mathrm{BS}}}$, and $\mathbf{n} \in \mathbb{C}^{N_{\mathrm{MS}} \times 1}$ is the noise vector modeled by the Gaussian mixture model [33-35]. At the receiver, a digital baseband decoder $\mathbf{W}_{\mathrm{BB}} \in \mathbb{C}^{N_{\mathrm{RF}} \times N_{\mathrm{S}}}$ and an analog decoder $\mathbf{W}_{\mathrm{RF}} \in \mathbb{C}^{N_{\mathrm{MS}} \times N_{\mathrm{RF}}}$ are applied to the received signal and the received signal after the decoding process can be shown as

$$
\mathbf{y}=\sqrt{\rho} \mathbf{W}_{\mathrm{BB}}^{H} \mathbf{W}_{\mathrm{RF}}^{H} \mathbf{H F}_{\mathrm{RF}} \mathbf{F}_{\mathrm{BB}} \mathbf{s}+\mathbf{W}_{\mathrm{BB}}^{H} \mathbf{W}_{\mathrm{RF}}^{H} \mathbf{n},
$$

where only phase shifters are used to design the analog RF precoders which have unit modulus constraint as $\left|\left(\mathbf{F}_{\mathrm{RF}}\right)_{i, l}\right|=\left|\left(\mathbf{W}_{\mathrm{RF}}\right)_{i, l}\right|=1$. Besides, the Middleton Class A noise [11] can be written as:

$$
\mathbf{n}=\mathbf{w}+\mathbf{z}
$$

where $\mathbf{w} \in \mathbb{C}^{N_{\mathrm{MS}} \times 1}$ denotes the additive white $\mathrm{GN}$ vector with an occurrence probability $1-\epsilon$ and $\mathbf{z} \in \mathbb{C}^{N_{\mathrm{MS}} \times 1}$ is IN vector that occurs with $\epsilon$ probability such that, $0 \leq \epsilon \leq 1$. The probability density function of the mixture model can be written as

$$
f=(1-\epsilon) N\left(0, \sigma_{w}^{2}\right)+\epsilon N\left(0, \sigma_{z}^{2}\right),
$$

where $N\left(0, \sigma_{w}^{2}\right)$ denotes the nominal Gaussian background noise with a variance $\sigma_{w}^{2}$ and $N\left(0, \sigma_{z}^{2}\right)$ represents IN component which is also distributed by Gaussian density with a variance $\sigma_{z}^{2}[30,11]$.

In this paper, due to the high free space path loss and limited scattering characteristics of mmWave, Saleh-Valenzuela [36] clustered channel model is used and thus, the channel matrix $\mathbf{H}$ is given by

$$
\mathbf{H}=\sqrt{\frac{N_{\mathrm{BS}} N_{\mathrm{MS}}}{N_{\mathrm{cl}} N_{\mathrm{ray}}}} \sum_{i=1}^{N_{\mathrm{cl}}} \sum_{l=1}^{N_{\mathrm{ray}}} \alpha_{i l}\left[\mathbf{a}_{\mathrm{MS}}\left(\phi_{i l}^{\mathrm{MS}}, \theta_{i l}^{\mathrm{MS}}\right) \mathbf{a}_{\mathrm{BS}}\left(\phi_{i l}^{\mathrm{BS}}, \theta_{i l}^{\mathrm{BS}}\right)^{H}\right],
$$

where $N_{\mathrm{cl}}$ and $N_{\text {ray }}$ denote the number of clusters and the number of rays respectively, $\alpha_{i l}$ refer to the complex gain of the channel with independent and identical (i.i.d) distribution $\alpha_{i l} \sim\left(0, \sigma^{2} \mathbf{I}\right)$ in which a normalization factor $\sum_{i=1}^{L} \sigma^{2} \mathbf{I}=\gamma$ is applied to the $\alpha_{i l}$ to ensure the constraint $\mathbb{E}\left[\|\mathbf{H}\|_{F}^{2}\right]=N_{\mathrm{BS}} N_{\mathrm{MS}}$. In addition, $\mathbf{a}_{\mathrm{MS}}\left(\phi_{i l}^{\mathrm{MS}}, \theta_{i l}^{\mathrm{MS}}\right)$ and $\mathbf{a}_{\mathrm{BS}}\left(\phi_{i l}^{\mathrm{BS}}, \theta_{i l}^{\mathrm{BS}}\right)^{H}$ represent the antenna array response vectors in the MS and BS respectively, while the azimuth (elevation) angles of arrival and departures are denoted as the coefficients $\phi_{i l}^{\mathrm{MS}}\left(\theta_{i l}^{\mathrm{MS}}\right)$ and $\phi_{i l}^{\mathrm{BS}}\left(\theta_{i l}^{\mathrm{BS}}\right)$. The array geometry is assumed as uniform square planar array and under this consideration, the array response vector at the BS can be defined as

$$
\mathbf{a}_{\mathrm{BS}}\left(\phi_{i l}^{\mathrm{BS}}, \theta_{i l}^{\mathrm{BS}}\right)=\frac{1}{\sqrt{N_{\mathrm{BS}}}}\left[\begin{array}{c}
1, \ldots, e^{j \frac{2 \pi}{\lambda} d\left(p \sin \left(\phi_{i l}^{\mathrm{BS}}\right) \sin \left(\theta_{i l}^{\mathrm{BS}}\right)+q \cos \left(\theta_{i l}^{\mathrm{BS}}\right)\right.}, \\
\ldots, e^{j\left(\sqrt{N_{B S}}-1\right) \frac{2 \pi}{\lambda} d\left(\sin \left(\phi_{i l}^{\mathrm{BS}}\right) \sin \left(\theta_{i l}^{\mathrm{BS}}\right)+\cos \left(\theta_{i l}^{\mathrm{BS}}\right)\right)}
\end{array}\right]^{T},
$$

where $\lambda$ and $d$ represent the wavelength of the signal and the space between antenna elements respectively, and $p$ and $q$ indicate the indices of the antennas such that, $0 \leq p \leq \sqrt{N_{\mathrm{BS}}}$ and $0 \leq q \leq$ $\sqrt{N_{\mathrm{BS}}}$. The array response vector at the MS $\mathbf{a}_{\mathrm{MS}}\left(\phi_{i l}^{\mathrm{MS}}, \theta_{i l}^{\mathrm{MS}}\right)$ can be defined using the same definition $[33,35,37]$.

\section{Proposed Adaptive Fuzzy Logic-Based Filter}

In conventional hybrid decoding systems, it is aimed to reduce the effects of noise and this noise is generally defined as Additive White Gaussian Noise (AWGN). However, for the mixture noise model, the IN components should be suppressed before passing the received signal through the decoder. Therefore, we propose an adaptive filter based on fuzzy logic that is added to the hybrid decoder as shown in Fig. 1 to suppress IN components at the receiver by reducing the effects of outlier amplitudes. Firstly, the median and the standard deviation of the real and imaginary parts of the received signal are calculated as 


$$
\begin{gathered}
\operatorname{med}^{R}=\operatorname{med}(|\operatorname{Re}\{\mathbf{r}\}|), \operatorname{med}^{I}=\operatorname{med}(|\operatorname{Im}\{\mathbf{r}\}|) \\
\operatorname{std}^{R}=\operatorname{std}(|\operatorname{Re}\{\mathbf{r}\}|), \operatorname{std}^{I}=\operatorname{std}(|\operatorname{Im}\{\mathbf{r}\}|),
\end{gathered}
$$

where the received signal vector can be shown in the form $\mathbf{r}=\left[r(1), r(2) \ldots, r\left(N_{\mathrm{MS}}\right)\right]$. After that, the real and imaginary parts of the corresponding statistic components are fed into the fuzzifier separately to find the Gaussian membership degrees using the following functions

$$
F^{R}(k)=\exp \left(\frac{-|\operatorname{Re}\{r(k)\}|-\left.m e d^{R}\right|^{2}}{2 s t d^{R}}\right) \text { and } F^{I}(k)=\exp \left(\frac{-|| \operatorname{Im}\{r(k)\}\left|-m e d^{I}\right|^{2}}{2 s t d^{I}}\right) .
$$

The filter is then applied to the received signal vector elementwise and the elements of the vector are redistributed depending on their fuzzy membership degrees. Thus, after the filtering operation, the elements of the received vector can be shown as

$$
r^{R}(k)=\operatorname{Re}\{r(k)\} \circ F^{R}(k) \text { and } r^{I}(k)=\operatorname{Im}\{r(k)\} \circ F^{I}(k),
$$

where $r^{R}(k)$ and $r^{I}(k)$ are the elements of the real and imaginary parts of the fuzzy filter output, respectively. Finally, $r^{R}(k)$ and $r^{I}(k)$ are combined to form the output of the fuzzy filter as

$$
r^{F}(k)=r^{R}(k)+j r^{I}(k)
$$

where it can be shown in the vector form as $\mathbf{r}^{F}=\left[r^{F}(1), r^{F}(2), \ldots, r^{F}\left(N_{\mathrm{MS}}\right)\right][29,30]$. After arranging the received signal, the output of the filter is passed through the hybrid combiners, $\mathbf{W}_{\mathrm{RF}}$ and $\mathbf{W}_{\mathrm{BB}}$, which are calculated using the alternating minimization method given in [38]. It should also be noted that the hybrid precoders, $\mathbf{F}_{\mathrm{RF}}$ and $\mathbf{F}_{\mathrm{BB}}$, are solved in the same way.

\subsection{Threshold Mechanism to Detect Outlier Samples}

In the proposed fuzzy filter, a threshold mechanism is designed to identify the outlier amplitudes which can be considered as IN samples, and the fuzzy filter is only applied to IN samples detected using the optimal threshold. Therefore, the proposed filter has the capability to perform adaptively in both GN and IN environments. Additionally, it is observed that the performance of the fuzzy filter is improved when the threshold is applied to the system. To detect IN samples, the modified Z-score is given by [32]

$$
M(k)=\frac{0.6745(r(k)-\operatorname{med}(\mathbf{r}))}{\mathrm{MAD}},
$$

where MAD denotes the Median Absolute Deviation that can be calculated as

$$
\operatorname{MAD}=\frac{1}{N_{\mathrm{MS}}} \sum_{k=1}^{N_{\mathrm{MS}}}|r(k)-\operatorname{med}(\mathbf{r})| .
$$

The potential outlier amplitudes can be detected when the absolute value of $M(k)$ is greater than 3.5 [32]. It is shown in the simulations that the optimal threshold can be selected as 3.5 and the samples are considered as IN for $|M(k)|>3.5$.

\section{Simulation Results}

In this section, we demonstrate the numerical simulation results of the proposed decoder with the adaptive fuzzy logic filter for different scenarios and give a comparison with the methods proposed in [25]. BER and the spectral efficiency performance of the system is calculated using the channel model represented in (5) for $N_{\mathrm{cl}}=10$ and $N_{\text {ray }}=5$. It is assumed that the perfect channel state information is known and the arrival and departure angles are assumed to have a uniform distribution in $[0,2 \pi]$, while the angular spread is selected as 10 . In addition, each cluster is organized to have unit average power as $\sigma_{\alpha, i}^{2}=1$. The simulations are executed in MATLAB for a BS with $N_{\mathrm{BS}}=144$ antennas which transmits $N_{\mathrm{S}}=1$ symbols with 256 packets to a MS with $N_{\mathrm{MS}}=36$ antennas through $N_{\mathrm{RF}}=6 \mathrm{RF}$ chains. Besides, the signal is modulated using 16-QAM with a carrier frequency of $28 \mathrm{GHz}$ and Signal to Impulsive Noise Ratio (SINR) is selected as -10 dB for IN channels. For more accurate results, each simulation is averaged over 1000 independent realizations 


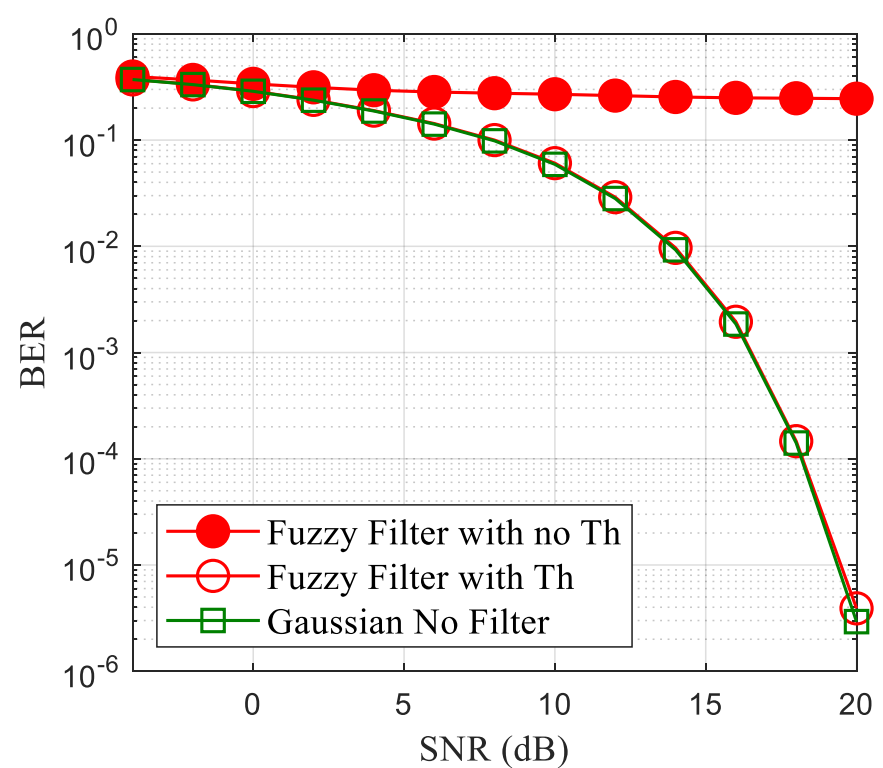

Fig. 2. BER versus SNR in Gaussian channel $(\epsilon=0)$ for Fuzzy filter with threshold and without threshold.

In Fig. 2, a fuzzy filter is applied to the system in the Gaussian channel where $\epsilon=0$ and BER performance is calculated for different Signal to Noise Ratio (SNR) values. The results show that the performance of the fuzzy filter is very poor when there is no threshold applied to the system and there is a huge gap with the performance of the system without fuzzy filter which is served as a benchmark. Therefore, there is a need to identify IN samples using the threshold mechanism as suggested in section 3. Fig. 3 plots the BER performance of the fuzzy filter for different threshold values at $6 \mathrm{~dB}$ SNR. It is observed that the optimal threshold can be selected as 3.5 and the optimal selection of the threshold makes the system suitable for both GN and IN conditions. The system is performing almost identical to the benchmark with the optimal threshold in the Gaussian channel as shown in Fig. 2.

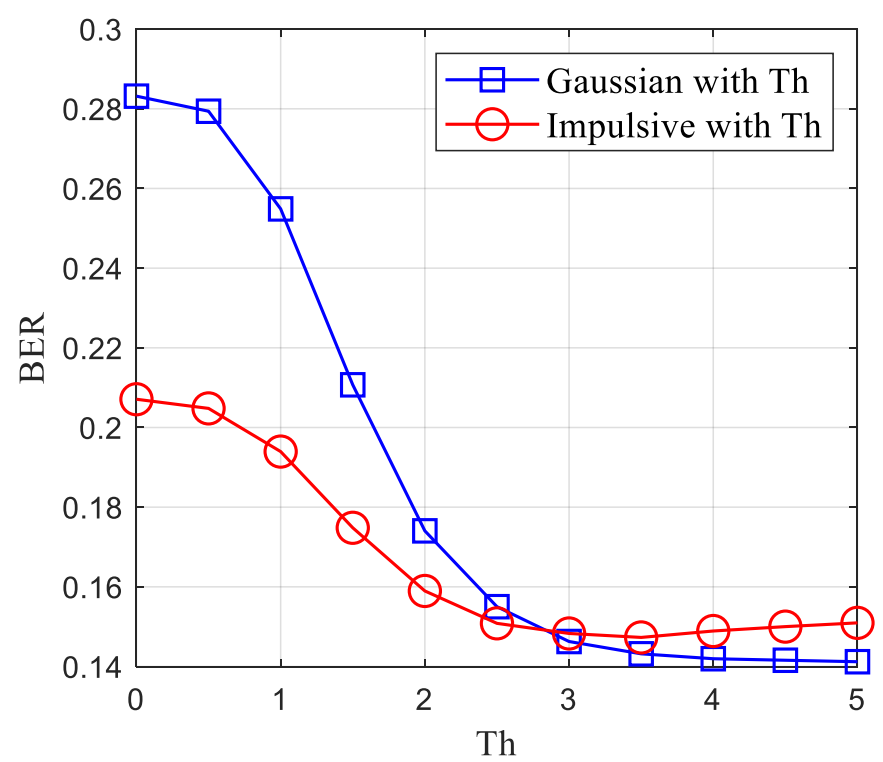

Fig. 3. BER versus noise threshold in Gaussian channel $(\epsilon=0)$ and IN channel $(\epsilon=0.02)$.

Fig. 4 plots the BER performance of the system with various SNR values in IN channel for $\epsilon=0.02$ and $\epsilon=0.04$. Here, it can be seen that the performance of the fuzzy filter with the threshold mechanism is better than the blanking and clipping filters for each IN channel. The clipping filter is performing the worst among the competing methods and the performance is very weak when there is no impulsive filter applied to the system. Moreover, Fig. 5 simulates the BER with respect to different epsilon values for SNR values $5 \mathrm{~dB}, 10 \mathrm{~dB}$, and $20 \mathrm{~dB}$. The results indicate that the performance of the fuzzy logic filter 
is the best among the competing methods for each epsilon and SNR values. The gap between the proposed method and the competing methods is increasing for low epsilon values at $20 \mathrm{~dB}$.

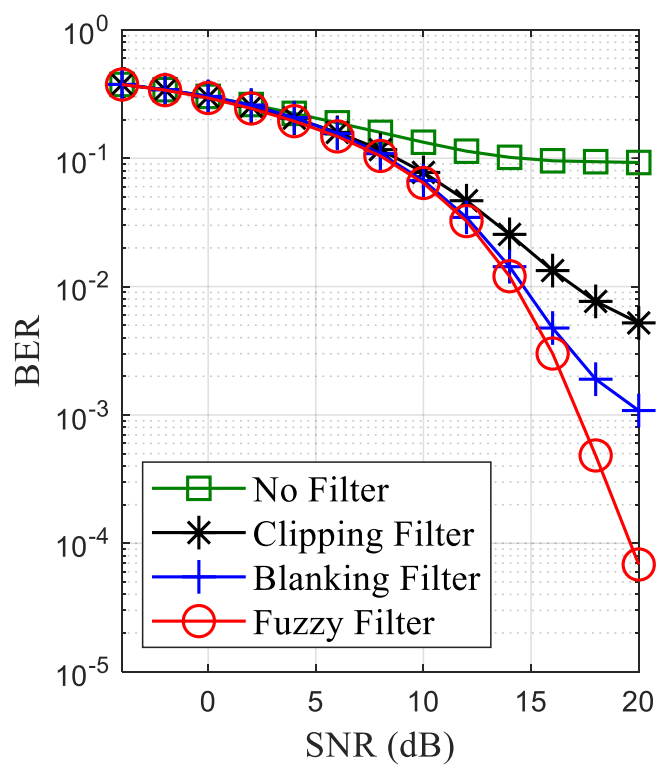

(a)

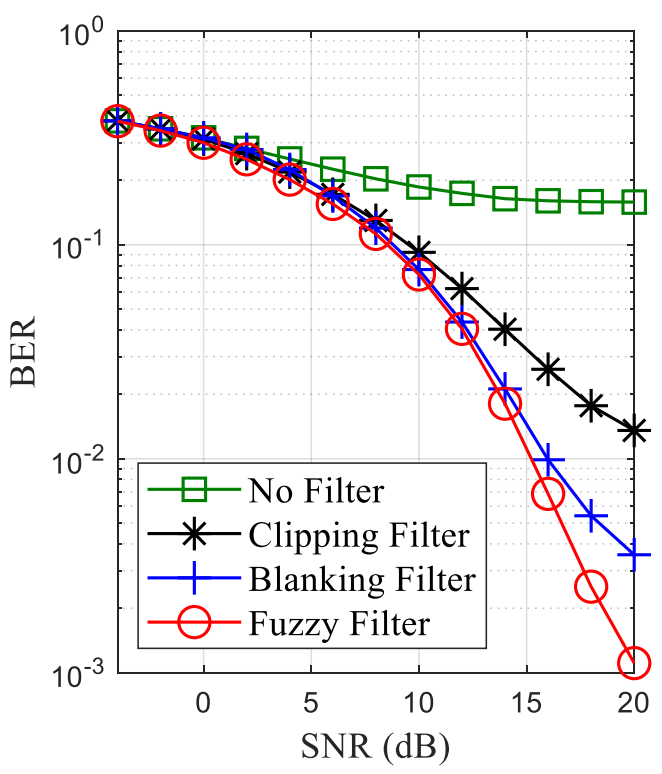

(b)

Fig. 4. BER versus SNR in IN channel (a) $\epsilon=0.02$ and (b) $\epsilon=0.04$

In Fig. 6, the spectral efficiency performance of the system is evaluated for different SNR values for $\epsilon=0.02$ and $\epsilon=0.04$, and the following rate expression is used to calculate the spectral efficiency [39]:

$$
R=\log _{2} \operatorname{det}\left(\mathbf{I}_{N_{\mathrm{s}}}+\frac{\rho}{N_{s}} \mathbf{R}_{n}^{-1} \mathbf{W}_{\mathrm{BB}}^{H} \mathbf{W}_{\mathrm{RF}}^{H} \mathbf{H} \mathbf{F}_{\mathrm{RF}} \mathbf{F}_{\mathrm{BB}} \mathbf{F}_{\mathrm{BB}}^{H} \mathbf{F}_{\mathrm{RF}}^{H} \mathbf{H}^{H}\left(\mathbf{W}_{\mathrm{RF}} \mathbf{W}_{\mathrm{BB}}\right)\right),
$$

where $\mathbf{R}_{n}=\sigma_{n}^{2} \mathbf{W}_{\mathrm{BB}}^{H} \mathbf{W}_{\mathrm{RF}}^{H} \mathbf{W}_{\mathrm{RF}} \mathbf{W}_{\mathrm{BB}}$. It is shown that the proposed method is performing better than the competing methods and a tremendous improvement is achieved when it is compared with the system without any IN filter implementation.

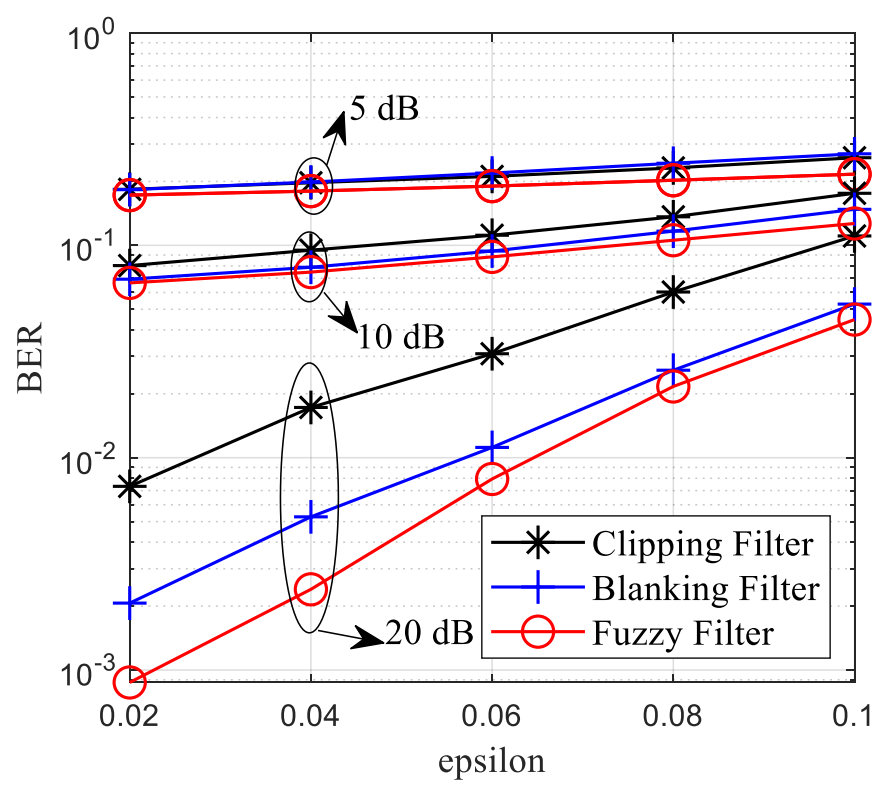

Fig. 5. BER versus epsilon for SNR values $5 \mathrm{~dB}, 10 \mathrm{~dB}$, and $20 \mathrm{~dB}$. 


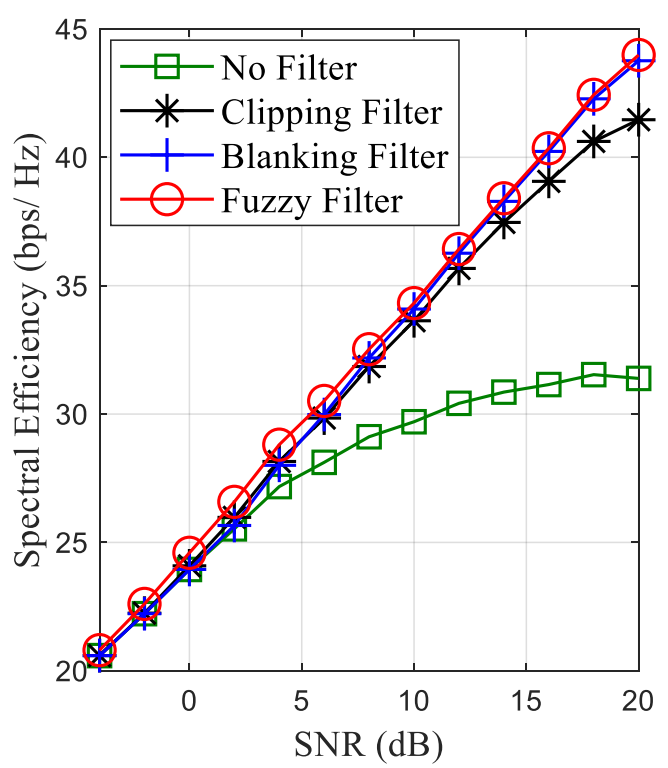

(a)

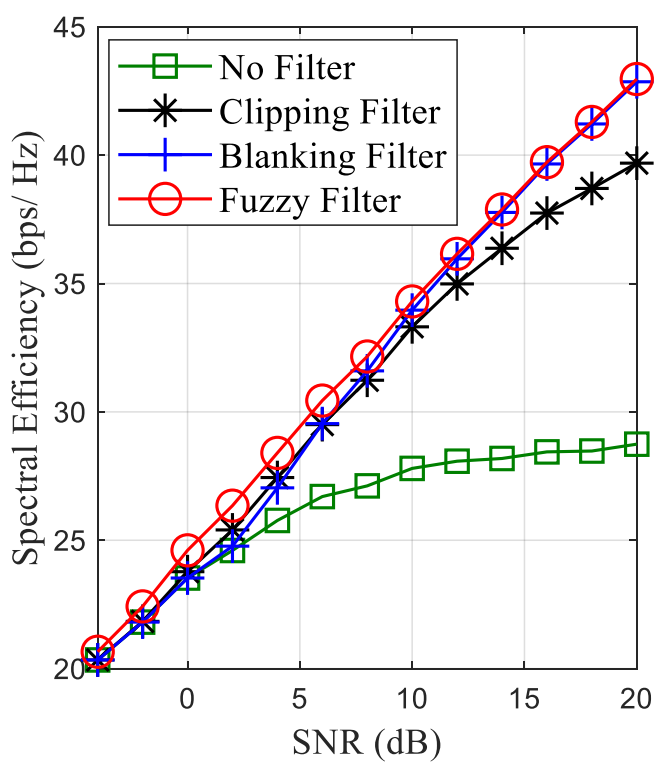

(b)

Fig. 6. Spectral efficiency versus SNR in IN channel (a) $\epsilon=0.02$ and (b) $\epsilon=0.04$.

\section{Conclusion}

In this paper, we built a novel approach to suppress IN noise in mmWave massive MIMO systems. The proposed filter is based on fuzzy logic and it is placed at the receiver to detect the outlier samples which can be considered as IN amplitudes. In addition, a threshold mechanism is designed to make the system suitable for the GN and IN environments. BER and spectral efficiency of the proposed and the competing methods are evaluated for different setups to provide a comparison. The simulation results illustrate that the proposed method has a better performance for detecting and eliminating IN components than the blanking and clipping filters. Furthermore, it can be seen clearly that the performance is improved enormously when it is compared with the results performed by the system without any IN filter applied.

\section{References}

[1] M. H. Alsharif and R. Nordin, "Evolution towards fifth generation (5G) wireless networks: Current trends and challenges in the deployment of millimetre wave, massive MIMO, and small cells," Telecommun. Syst., vol. 64, no. 4, pp. 617-637, 2017, doi: 10.1007/s11235-016-0195$\mathrm{X}$.

[2] M. Shariat et al., "5G radio access above 6 GHz," Trans. Emerg. Telecommun. Technol., 2016, doi: 10.1002/ett.3076.

[3] L. Su, Y. Niu, A. V. Vasilakos, Y. Li, and D. Jin, "A survey of millimeter wave communications (mmWave) for 5G: opportunities and challenges," Wirel. Networks, vol. 21, no. 8, pp. 26572676, 2015, doi: 10.1007/s11276-015-0942-z.

[4] T. S. Rappaport et al., "Millimeter Wave Mobile Communications for 5G Cellular: It Will Work!," IEEE Access, vol. 1, pp. 335-349, 2013, doi: 10.1109/ACCESS.2013.2260813.

[5] Z. Pi and F. Khan, "An introduction to millimeter-wave mobile broadband systems," IEEE Commun. Mag., vol. 49, no. 6, pp. 101-107, 2011, doi: 10.1109/MCOM.2011.5783993.

[6] M. Vu and A. Paulraj, "MIMO wireless linear precoding," IEEE Signal Process. Mag., vol. 24, no. 5, pp. 86-105, 2007, doi: 10.1109/MSP.2007.904811.

[7] A. Alkhateeb, J. Mo, N. González-Prelcic, and R. W. Heath, "MIMO precoding and combining solutions for millimeter-wave systems," IEEE Commun. Mag., 2014, doi: 10.1109/MCOM.2014.6979963.

[8] O. El Ayach, S. Rajagopal, S. Abu-Surra, Z. Pi, and R. W. Heath, "Spatially sparse precoding in millimeter wave MIMO systems," IEEE Trans. Wirel. Commun., vol. 13, no. 3, pp. 14991513, 2014, doi: 10.1109/TWC.2014.011714.130846. 
[9] B. Q. Vuong, H. T. Huynh, and H. N. Do, "Monte-Carlo performance analysis of OFDM system in the presence of multi-path fading environment and non-Gaussian noise," in International Conference on Recent Advances in Signal Processing, Telecommunications \& Computing (SigTelCom), 2018, doi: 10.1109/SIGTELCOM.2018.8325796

[10] D. Middleton, An introduction to: Statistical communication theory, Wiley-IEEE Press, 2009.

[11] X. Wang and H. V. Poor, "Robust multiuser detection in non-gaussian channels," IEEE Trans. Signal Process., 1999, doi: 10.1109/78.740103.

[12] F. Rouissi, A. J. H. Vinck, and A. Ghazel, "On the simulation of the Middleton Class-A noise model for single- and multi-carrier modulation in power line communication," Telecommun. Syst., no. 0123456789, 2021, doi: 10.1007/s11235-020-00746-x.

[13] M. S. Alam, B. Selim, and G. Kaddoum, "Analysis and Comparison of Several Mitigation Techniques for Middleton Class-A Noise," in IEEE Latin-American Conference on Communications (LATINCOM), 2019, doi: 10.1109/LATINCOM48065.2019.8938020

[14] K. Hagglund and E. Axell, "Adaptive demodulation in class a impulse noise channels," in IEEE Conference and Exhibition on Global Telecommunications (GLOBECOM), 2019, doi: 10.1109/GLOBECOM38437.2019.9013968

[15] S. Moaveninejad, A. Kumar, M. Elgenedy, M. Magarini, N. Al-Dhahir, and A. M. Tonello, "Gaussian-Middleton Classification of Cyclostationary Correlated Noise in Hybrid MIMOOFDM WiNPLC," in IEEE International Conference on Communications (ICC), 2019, doi: 10.1109/ICC.2019.8761152.

[16] L. Liu and M. G. Amin, "Performance analysis of GPS receivers in non-Gaussian noise incorporating precorrelation filter and sampling rate," IEEE Trans. Signal Process., 2008, doi: 10.1109/TSP.2006.890827.

[17] K. Maeda, H. Nakata, and K. Fujito, "Analysis of BER of 16QAM signal in AM/16QAM hybrid optical transmission system," Electron. Lett., vol. 29, no. 7, pp. 640-642, 1993.

[18] R. I. P, "RECOMMENDATION ITU-R P.372-9 Radio noise," Group, 2007.

[19] V. Katkovnik, "New concept of adaptive beamforming for moving sources and impulse noise environment," Signal Processing, 2000, doi: 10.1016/S0165-1684(00)00094-3.

[20] M. S. Lee, V. Katkovnik, and Y. H. Kim, "Robust approximate median beamforming for phased array radar with antenna switching," Signal Processing, 2004, doi: 10.1016/j.sigpro.2004.05.007.

[21] H. Abu Hilal, "Error Rate Analysis of ZF and MMSE Decoders for Massive Multi Cell MIMO Systems in Impulsive Noise Channels," Int. J. Wirel. Inf. Networks, 2019, doi: 10.1007/s10776019-00422

[22] F. H. Juwono, R. Reine, and L. LIU, "Performance of impulsive noise blanking in precoded OFDM-based PLC systems," in IEEE International Conference on Communication Systems (ICCS), 2016, pp. 1-6, doi: 10.1109/ICCS.2016.7833562.

[23] K. M. Rabie and E. Alsusa, "Performance analysis of adaptive hybrid nonlinear preprocessors for impulsive noise mitigation over power-line channels," in International Conference on Communications (ICC), 2015, pp. 728-733, doi: 10.1109/ ICC.2015. 7248408

[24] F. Sarabchi and C. Nerguizian, "Impulsive noise mitigation for OFDM-based systems using enhanced blanking nonlinearity," in IEEE 25th Annual International Symposium on Personal, Indoor, and Mobile RadioCommunication (PIMRC), 2014, p.841-845, doi: 10.1109/PIMRC.2014.7136282.

[25] L. Shhab, A. Rizaner, A. H. Ulusoy, and H. Amca, "Suppressing the effect of impulsive noise on millimeter-wave communications systems," Radioengineering, 2020, doi: 10.13164/RE.2020.0376.

[26] H. Abu Hilal, "Neural networks applications for CDMA systems in non-Gaussian multi-path channels," AEU - Int. J. Electron. Commun., vol. 73, pp. 150-156, 2017, doi: 10.1016/j.aeue.2017.01.006.

[27] R. Barazideh, S. Niknam, and B. Natarajan, "Impulsive noise detection in OFDM-based systems: A deep learning perspective," in IEEE Annual Computing and Communication Workshop and Conference (CCWC), 2019, doi: 10.1109/CCWC.2019.8666489. 
[28] O. Delgado and F. Labeau, "Deep Learning Decoder for MIMO Communications with Impulsive Noise," in IEEE Consumer Communications and Networking Conference (CCNC), 2020, doi: 10.1109/CCNC46108.2020.9045329.

[29] A. M. Hmidat, B. S. Sharif, and W. L. Woo, "Fuzzy decorrelating detector for non-Gaussian CDMA channel," Electron. Lett., 2004, doi: 10.1049/el:20040595.

[30] A. H. Ulusoy and A. Rizaner, "Adaptive path selective fuzzy decorrelating detector under impulsive noise for multipath fading CDMA systems," IEEE Commun. Lett., 2008, doi: 10.1109/LCOMM.2008.072012.

[31] A. H. Ulusoy and A. Rizaner, "RBF network assisted adaptive path selective decorrelating detector under impulsive noise for multipath fading CDMA systems," Ann. des Telecommun. 2011, doi: 10.1007/s12243-010-0212-0.

[32] T. Crosby, B. Iglewicz, and D. C. Hoaglin, "How to Detect and Handle Outliers," Technometrics, 1994, doi: 10.2307/1269377.

[33] A. Alkhateeb, G. Leus, and R. W. Heath, "Limited Feedback Hybrid Precoding for Multi-User Millimeter Wave Systems," IEEE Trans. Wirel. Commun., 2015, doi: 10.1109/TWC.2015.2455980.

[34] A. Alkhateeb, O. El Ayach, G. Leus, and R. W. Heath, "Channel estimation and hybrid precoding for millimeter wave cellular systems," IEEE J. Sel. Top. Signal Process., 2014, doi: 10.1109/JSTSP.2014.2334278.

[35] X. Yu, J. C. Shen, J. Zhang, and K. B. Letaief, "Alternating Minimization Algorithms for Hybrid Precoding in Millimeter Wave MIMO Systems," IEEE J. Sel. Top. Signal Process., vol. 10, no. 3, pp. 485-500, 2016, doi: 10.1109/JSTSP.2016.2523903..

[36] A. A. M. Saleh and R. A. Valenzuela, "A Statistical Model for Indoor Multipath Propagation," IEEE J. Sel. Areas Commun., 1987, doi: 10.1109/JSAC.1987.1146527.

[37] V. Raghavan and A. M. Sayeed, "Sublinear capacity scaling laws for sparse MIMO channels," IEEE Trans. Inf. Theory, 2011, doi: 10.1109/TIT.2010.2090255.

[38] M. Mulla, A. H. Ulusoy, A. Rizaner, and H. Amca, "Barzilai-Borwein Gradient Algorithm Based Alternating Minimization for Single User Millimeter Wave Systems," IEEE Wirel. Commun. Lett., 2020, doi: 10.1109/LWC.2019.2960691.

[39] C. Rusu, R. Mèndez-Rial, N. González-Prelcic, and R. W. Heath, "Low complexity hybrid precoding strategies for millimeter wave communication systems,"IEEE Trans. Wireless Commun.,vol.15,no.12,383pp.8380-8393,Dec. 2016, doi:10.1109//TWC.2016.2614495 
Figures
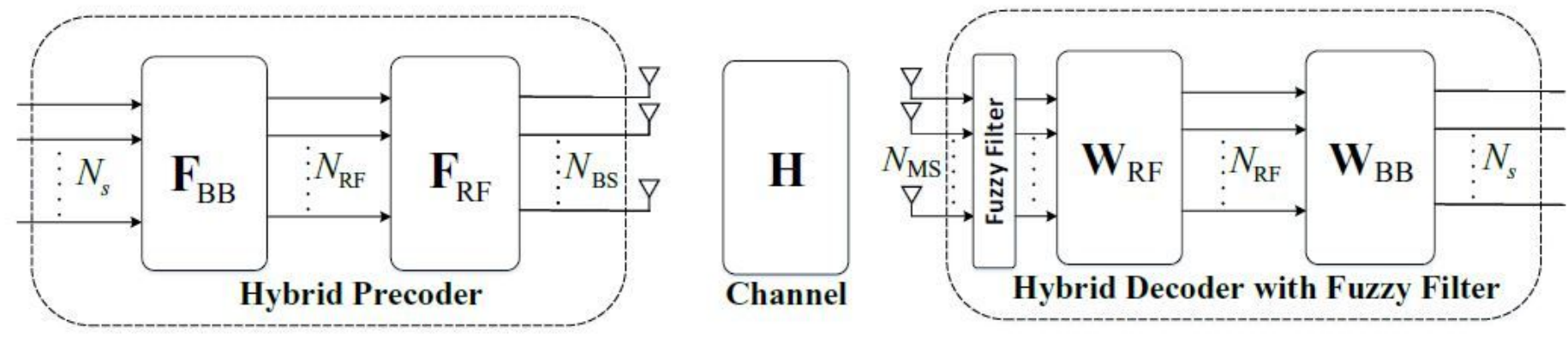

Figure 1

System model for hybrid analog/digital architecture with a fuzzy filter in single-user mmWave massive MIMO system.

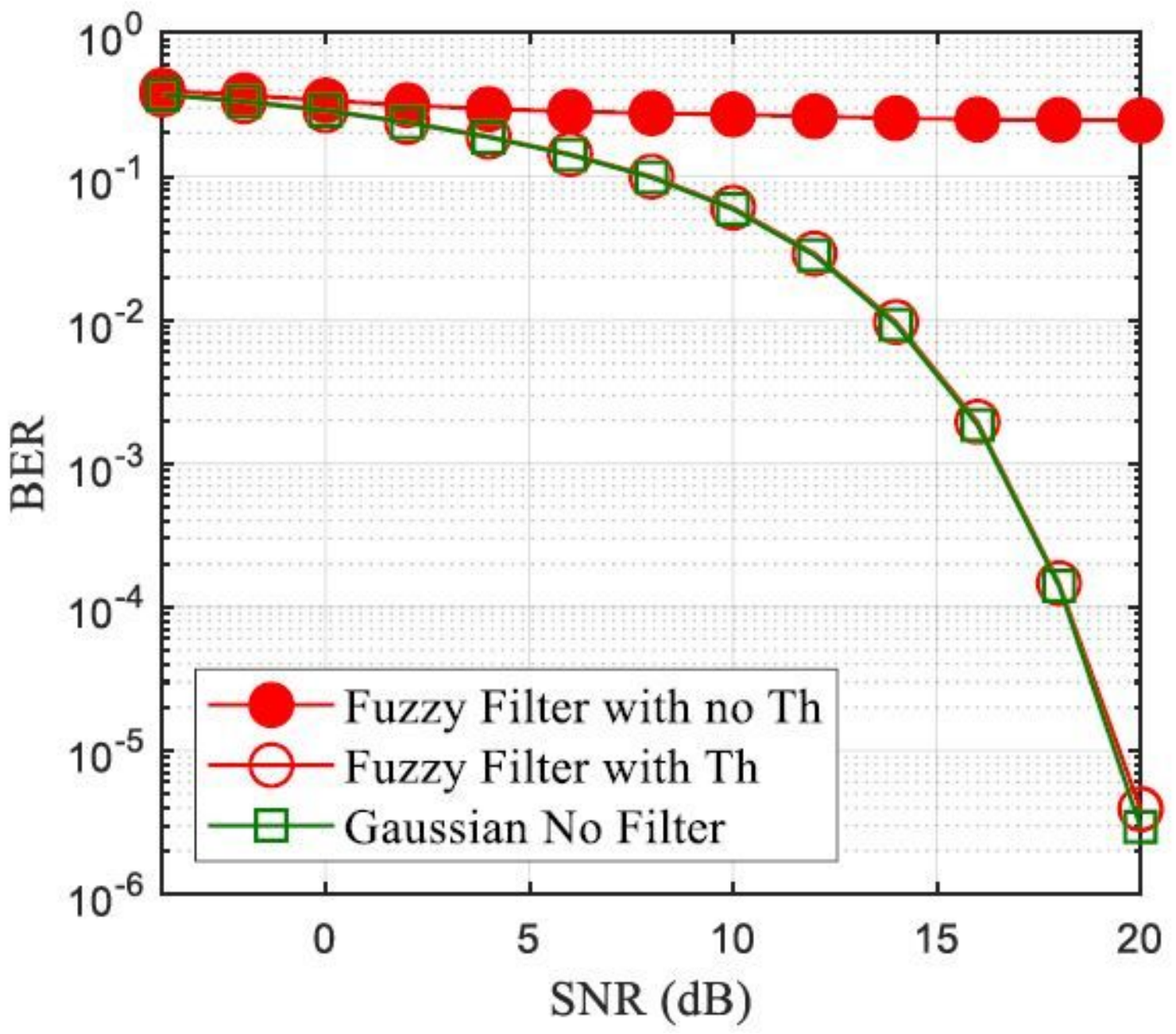

Figure 2

BER versus SNR in Gaussian channel $(\mathbb{Q}=0)$ for Fuzzy filter with threshold and without threshold. 




Figure 3

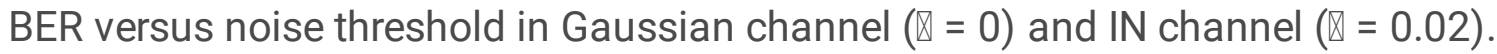






(a)

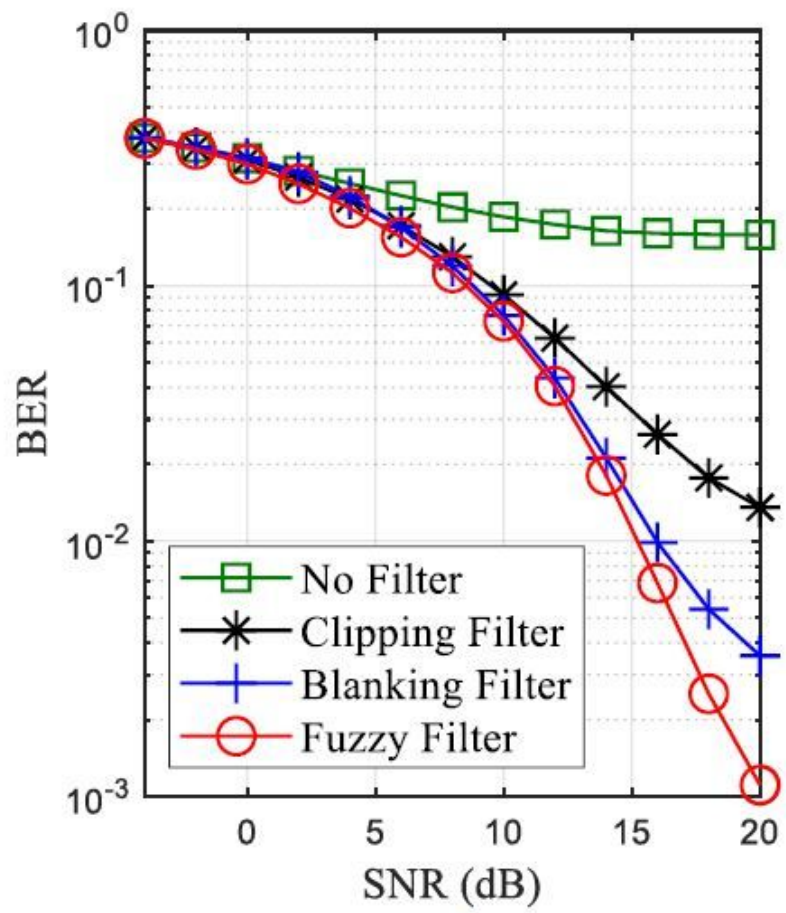

(b)

Figure 4

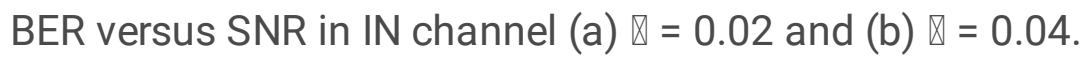




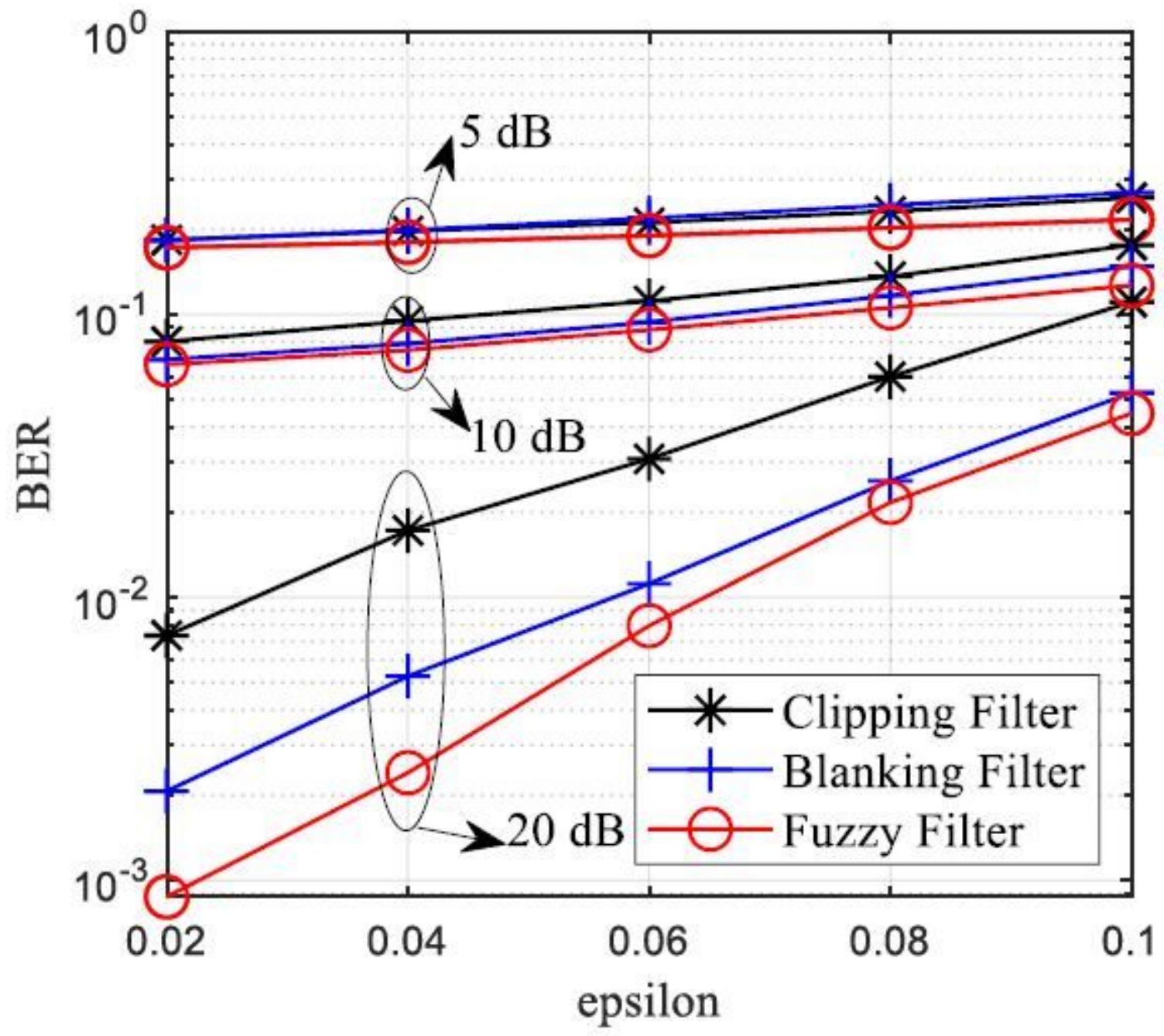

Figure 5

BER versus epsilon for SNR values $5 \mathrm{~dB}, 10 \mathrm{~dB}$, and $20 \mathrm{~dB}$. 


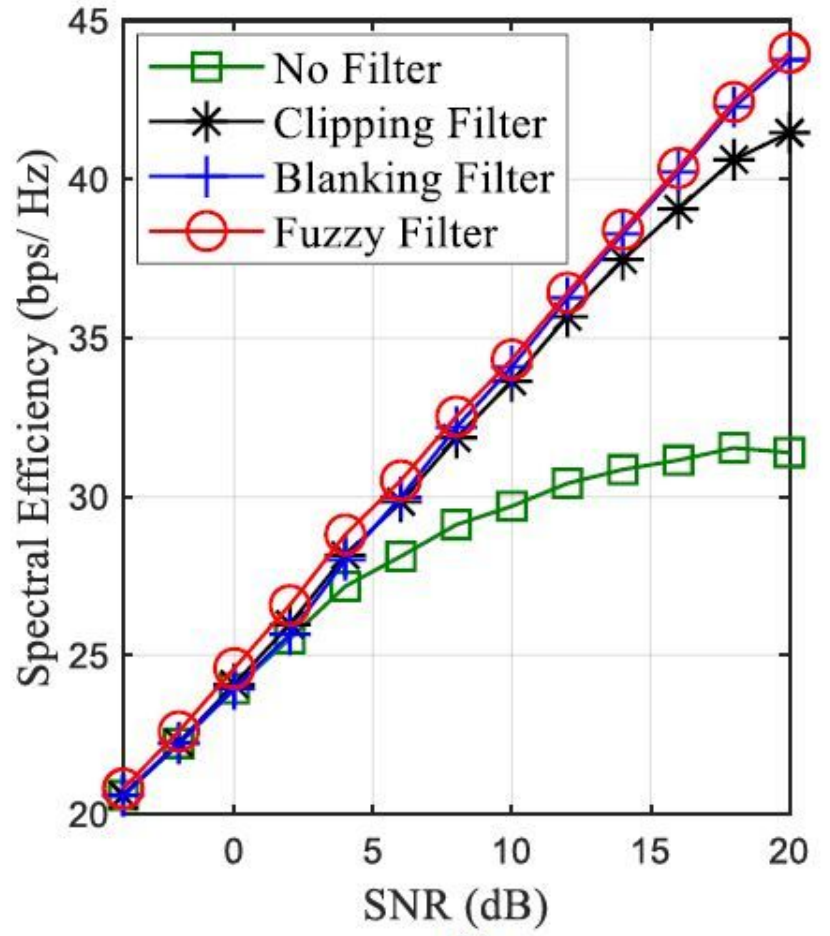

(a)

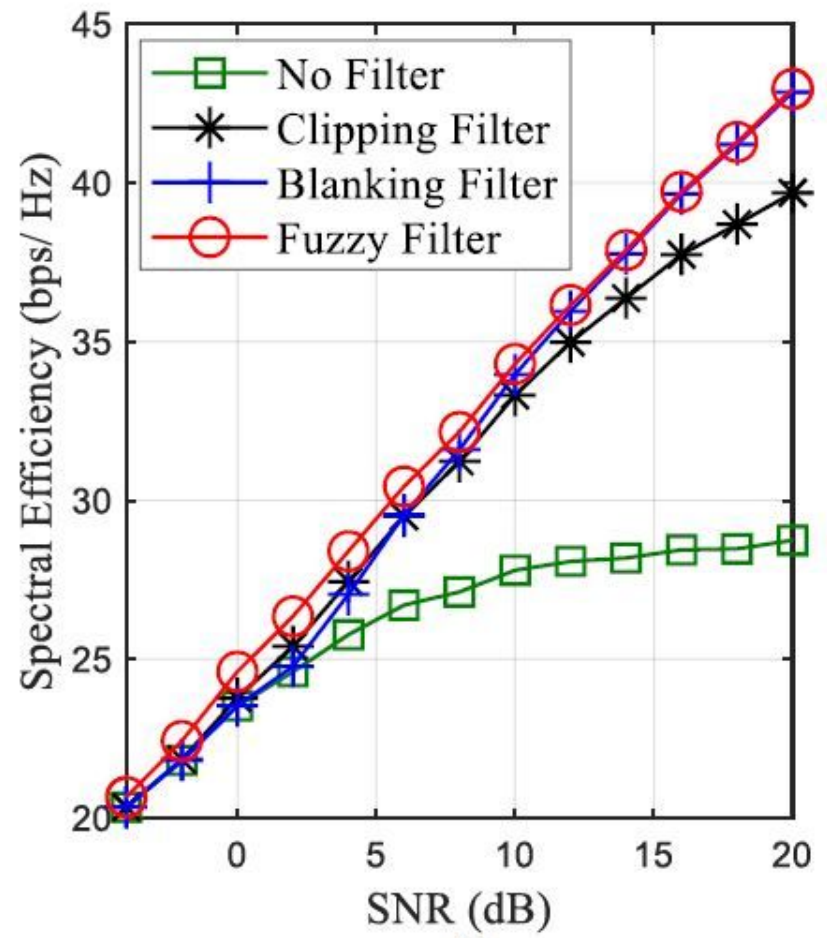

(b)

Figure 6

Spectral efficiency versus SNR in IN channel $(a) \otimes=0.02$ and $(b) \otimes=0.04$. 